\title{
Sedation-related complications during anesthesiologist-administered sedation for endoscopic retrograde cholangiopancreatography: a prospective study
}

Chengcheng C. Zhang ${ }^{1 *}$ (D) Nicole Ganion², Phillip Knebel ${ }^{3}$, Christian Bopp ${ }^{4}$, Thorsten Brenner², Markus A. Weigand ${ }^{2}$, Peter Sauer ${ }^{1}$ and Anja Schaible ${ }^{3}$

\begin{abstract}
Background: Patients undergoing endoscopic retrograde cholangiopancreatography (ERCP) require adequate sedation or general anesthesia. To date, there is lack of consensus regarding who should administer sedation in these patients. Several studies have investigated the safety and efficacy of non-anesthesiologist-administered sedation for ERCP; however, data regarding anesthesiologist-administered sedation remain limited. This prospective single-center study investigated the safety and efficacy of anesthesiologist-administered sedation and the rate of successful performed ERCP procedures.

Methods: The study included 200 patients who underwent ERCP following anesthesiologist-administered sedation with propofol and remifentanil. Procedural data, oxygen saturation, systolic blood pressure (SBP), heart rate, recovery score, patient and endoscopist satisfaction, as well as 30-day mortality and morbidity data were analyzed.

Results: Sedation-related complications occurred in 36 of 200 patients (18\%) and included hypotension (SBP $<90 \mathrm{mmHg})$ and hypoxemia $\left(\mathrm{O}_{2}\right.$ saturation $\left.<90 \%\right)$ in 18 patients $(9 \%)$ each. Most events were minor and did not necessitate discontinuation of the procedure. However, ERCP was terminated in 2 patients (1\%) secondary to sedation-related complications. Successful cannulation was performed in all patients. The mean duration of the examination was $25 \pm 16 \mathrm{~min}$. Mean recovery time was $14 \pm 10 \mathrm{~min}$, and high post-procedural satisfaction was observed in both, patients (mean visual analogue scale [VAS] 9.6 \pm 0.8) and endoscopists (mean VAS 9.3 \pm 1.3 ).
\end{abstract}

Conclusion: This study suggests that anesthesiologist-administered sedation is safe in patients undergoing ERCP and is associated with a high rate of successful ERCP, shorter procedure time, and more rapid post-anesthesia recovery, with high patient and endoscopist satisfaction.

Keywords: Anesthesia, Hypotension, Hypoxemia, ERCP, Endoscopic retrograde cholangiopancreatography, Sedation

\footnotetext{
* Correspondence: christine.zhang@med.uni-heidelberg.de

'Department of Gastroenterology, Heidelberg University Hospital, Im

Neuenheimer Feld 410, 69120 Heidelberg, Germany

Full list of author information is available at the end of the article
}

\section{$\triangle B M C$}

(c) The Author(s). 2020 Open Access This article is licensed under a Creative Commons Attribution 4.0 International License, which permits use, sharing, adaptation, distribution and reproduction in any medium or format, as long as you give appropriate credit to the original author(s) and the source, provide a link to the Creative Commons licence, and indicate if changes were made. The images or other third party material in this article are included in the article's Creative Commons licence, unless indicated otherwise in a credit line to the material. If material is not included in the article's Creative Commons licence and your intended use is not permitted by statutory regulation or exceeds the permitted use, you will need to obtain permission directly from the copyright holder. To view a copy of this licence, visit http://creativecommons.org/licenses/by/4.0/ The Creative Commons Public Domain Dedication waiver (http://creativecommons.org/publicdomain/zero/1.0/) applies to the data made available in this article, unless otherwise stated in a credit line to the data. 


\section{Background}

Endoscopic retrograde cholangiopancreatography (ERCP) is a complex and time-consuming procedure necessitating adequate sedation or general anesthesia. Reportedly, complication and mortality rates associated with ERCP are 5-10\% and $0.1-1 \%$, respectively [1-5]. Complications of ERCP include acute pancreatitis, bleeding, and perforation [1].

To date, optimal sedation techniques for complex endoscopic procedures remain unclear. There is lack of global consensus regarding the choice of practitioners to administer sedation and the optimal sedation technique for ERCP. In some countries (e.g., France), sedation is performed only by anesthesiologists. However, the German 'Update S3-guideline: sedation for gastrointestinal endoscopy 2014' clearly defines and summarizes the staff and technical requirements as follows [6]: Sedation can be administered by a trained nurse under a physician's supervision during simple endoscopic examinations. A second physician with experience in intensive care medicine should be present in cases involving a high procedural risk and for those requiring prolonged complex endoscopic interventions. Anesthesiologistadministered sedation is necessary in high-risk patients (those categorized as American Society of Anesthesiologists [ASA] class III-IV, in those undergoing difficult endoscopic interventions or in those with complex anatomy predisposing to a high risk of airway obstruction).

All sedation techniques are associated with the risk of cardiopulmonary complications, such as hypoventilation, respiratory depression, apnea, hypotension, and bradycardia [7]. A Cochrane Review evaluated the efficacy and safety of sedation techniques for ERCP in adults [7]. The authors intended to compare complication rates between sedation performed by anesthesia- and non-anesthesia personnel. However, they could not identify relevant studies involving anesthesia personnel. Therefore, the authors analyzed the results of 4 randomized trials that compared midazolam and meperidine with propofolonly sedation in patients undergoing ERCP with sedation performed by non-anesthesia personnel. No significant differences were observed in cardiorespiratory complications, and no immediate mortality was reported. Patients receiving propofol-only sedation for ERCP showed more rapid post-anesthesia recovery than patients receiving midazolam and meperidine [7-11], and the former group also showed higher patient satisfaction $[7,9,10]$.

In all studies identified by the Cochrane Review, sedation was performed only by non-anesthesia personnel. Therefore, whether anesthesiologist-administered sedation affects sedation-related complications is still elusive [7]. To date, few studies have described ERCP using sedation administered by anesthesiologists. The role of anesthesia personnel in the administration of sedation and the effects of sedation administered by anesthesia personnel with regard to the safety profile and complication rates of sedation during complex endoscopic procedures remains unclear. This prospective cohort study investigated the efficacy and safety of anesthesiologistadministered sedation for ERCP.

\section{Methods}

\section{Study design}

Our study protocol conformed to the Declaration of Helsinki and was approved by the Institutional Ethics Committee vide letter no S-457/2013. Written informed consent was obtained from all patients included in the study.

This prospective single-center study was performed at the Interdisciplinary Endoscopy Center, University Hospital of Heidelberg and included all adults who underwent ERCP at this center between March 2014 and November 2014. During this study period, all ERCP procedures were performed using anesthesiologist-administered sedation with only propofol and remifentanil. Baseline patient characteristics (age, sex, height and weight) were recorded. ERCP was performed by three experienced specialists in interventional endoscopy.

\section{Study population}

Exclusion criteria were age $<18$ years, pregnancy, lack of informed consent (e.g. patients with mental retardation or language issues), a history of propofol and/or remifentanil allergy, baseline $\mathrm{O}_{2}$ saturation $\left(\mathrm{O}_{2}\right.$ sat $)<90 \%$, baseline systolic blood pressure (SBP) $<90 \mathrm{mmHg}$, and need for general anesthesia.

\section{Data collection}

Following evaluation, patients were categorized based on the ASA physical status classification. Patient monitoring during ERCP included clinical observation, non-invasive blood pressure measurement every $5 \mathrm{~min}$, continuous monitoring of $\mathrm{O}_{2}$ sat, heart rate and electrocardiography. Patient characteristics, cardiorespiratory and procedural data, sedation-related events, and patient and endoscopist satisfaction data were recorded in a case report format before, during, and after examination.

All patients were transferred to a recovery unit and monitored by a nurse after ERCP. Patients were evaluated $30 \mathrm{~min}$ after termination of administration of sedation for post-anesthesia recovery using the "Post Anesthesia Recovery Score" (PARS), which evaluates patients' status with regard to the following criteria: activity (able to move all 4 extremities voluntarily or on command [2 points], able to move 2 extremities voluntarily or on command [ 1 point], unable to move extremities voluntarily or on command [0 points]), consciousness (fully awake [2 points], arousable on calling [ 1 point], unresponsive [0 points]), circulation (SBP $\pm 20 \%$ of pre-anesthetic level [2 points], 
$\mathrm{SBP} \pm 20-49 \%$ of pre-anesthetic level [ 1 point], $\mathrm{SBP} \pm 50 \%$ of pre-anesthetic level $[0$ points]), respiration (able to breathe deeply and cough freely [2 points], dyspnea or limited breathing [1 point], apneic [0 points]), and color (normal [2 points], pale, dusky, blotchy, jaundiced, or other [ 1 point], cyanotic [0 points]) [12]. Complete recovery was defined as a maximum score of 10 points. Patients were discharged from the recovery unit after fully recovered. Furthermore, after regaining full consciousness, all patients filled a questionnaire and a 6-point Likert scale (1: very satisfied, and 6: very dissatisfied) regarding patient satisfaction and readiness to undergo a repeat examination under the same conditions. Endoscopist satisfaction was also recorded using a 6-point Likert scale.

After the procedure, patients were followed-up for 30 days to assess complications, as well as morbidity and mortality. Post-ERCP pancreatitis was defined as acute onset of epigastric pain, elevated serum lipase/amylase levels (at least 3-fold higher than the upper limit of normal), and characteristic imaging findings. Patients with at least 2 of these 3 criteria were diagnosed with post-ERCP pancreatitis. According to the Atlanta classification system, severity of pancreatitis was categorized as mild pancreatitis (absence of organ failure and local or systemic complications), moderate pancreatitis (transient organ failure resolving within $48 \mathrm{~h}$ and/or local or systemic complications without persistent organ failure), and severe pancreatitis (organ failure persisting $>48 \mathrm{~h}$ ) $[13,14]$.

\section{Sedation techniques}

All patients received continuous oxygen supplementation during the procedure at the rate of $41 / \mathrm{min}$ via nasal cannula. Xylocaine spray (3 jets) was used for oropharyngeal anesthesia without any other oral premedication. According to the German 'Update S3-guideline: sedation for gastrointestinal endoscopy 2014' an initial propofol loading dose of $40-60 \mathrm{mg}$ (depending on age, body weight and comorbidities of the patient) was administered through an intravenous catheter for sedation followed by bodyweight adapted continuous infusion of propofol (1.5-4.5 $\mathrm{mg} / \mathrm{kg} / \mathrm{hour}$ ) and remifentanil $(0.025-0.2 \mu \mathrm{g} / \mathrm{kg} / \mathrm{min})$ [6]. An additional propofol bolus $(10-20 \mathrm{mg})$ was injected in patients showing signs of discomfort (e.g. agitation, uncontrolled movements, facial expressions and sounds).

\section{Primary and secondary endpoints}

Primary endpoints of this study included sedationrelated complications, i.e., hypoxemic events (defined as desaturation represented by $\mathrm{O}_{2}$ sat $<90 \%$ for at least 2 $\mathrm{min}$ ), hypotension (defined as SBP $<90 \mathrm{mmHg}$ ), bradycardia (defined as heart rate $<40$ beats per min $[\mathrm{bpm}]$ ), failure to complete ERCP secondary to sedation-related complications, 30-day mortality secondary to sedation, anesthesia or ERCP-related complications. Secondary endpoints included endoscopist and patient satisfaction, patients' willingness to undergo a repeat examination under the same conditions, deep cannulation rate, and time until intubation (defined as time between insertion of the endoscope through the pharynx and intubation of the major papilla), rate of successful ERCP, duration of the procedure (defined as time between insertion of the endoscope through the pharynx and removal of the endoscope from the pharynx), cumulative doses of propofol $(\mathrm{mg})$ and remifentanil $(\mu \mathrm{g})$, time until recovery after sedation (defined as time between termination of the procedure and transfer to recovery unit).

\section{Statistical analysis}

Descriptive statistics were used for all parameters. Results are expressed as means \pm standard deviation and ranges for continuous variables and numbers and percentages for categorical variables. Values recorded for the 6-point Likert-scale (1: very satisfied and 6: very dissatisfied) were converted into a 10-point visual analogue scale (VAS) (0: very dissatisfied and 10: very satisfied) by linear transformation. All data were analyzed using the IBM SPSS Statistics 24 software.

\section{Results}

During the study period, 345 patients underwent ERCP and were assessed for eligibility to be included in the study (Fig. 1). As shown in Fig. 1 and Table 1, 136 patients could not be enrolled owing to the following reasons: 43 patients refused to participate, 48 did not meet the inclusion criteria $\left(n=7\right.$ : baseline $\mathrm{O}_{2}$ sat $<90 \%, n=3$ : basal SBP $<90 \mathrm{mmHg}, n=30$ : lack of written informed consent for various reasons, $n=2$ : age $<18$ years, $n=6$ : need for general anesthesia), technical failure occurred in 4 patients, and 41 patients were excluded because they presented for ERCP on $\geq 2$ occasions during the study period. In these cases, patients' data were included in the statistical analysis only once. Eventually, 209 patients were enrolled. However, 8 patients were secondarily excluded because ERCP could not be performed owing to non-sedation related causes (7 patients were excluded because they underwent endoscopic ultrasonography or gastroscopy instead of ERCP, and 1 patient was excluded owing to an allergic reaction against the contrast agent administered during the ERCP). Therefore, 201 patients were included in the study. Notably, 1 patient who accidentally received additional drugs (different from the prescribed protocol) for sedation was excluded. After exclusion of patients owing to the aforementioned reasons, 200 patients were investigated.

Table 2 shows the baseline clinical and demographic characteristics of the investigated patients. Notably, the study included $64 \%$ men $(n=128 / 200)$, and the age of the study population was $19-89$ years (mean 56.3 years). 


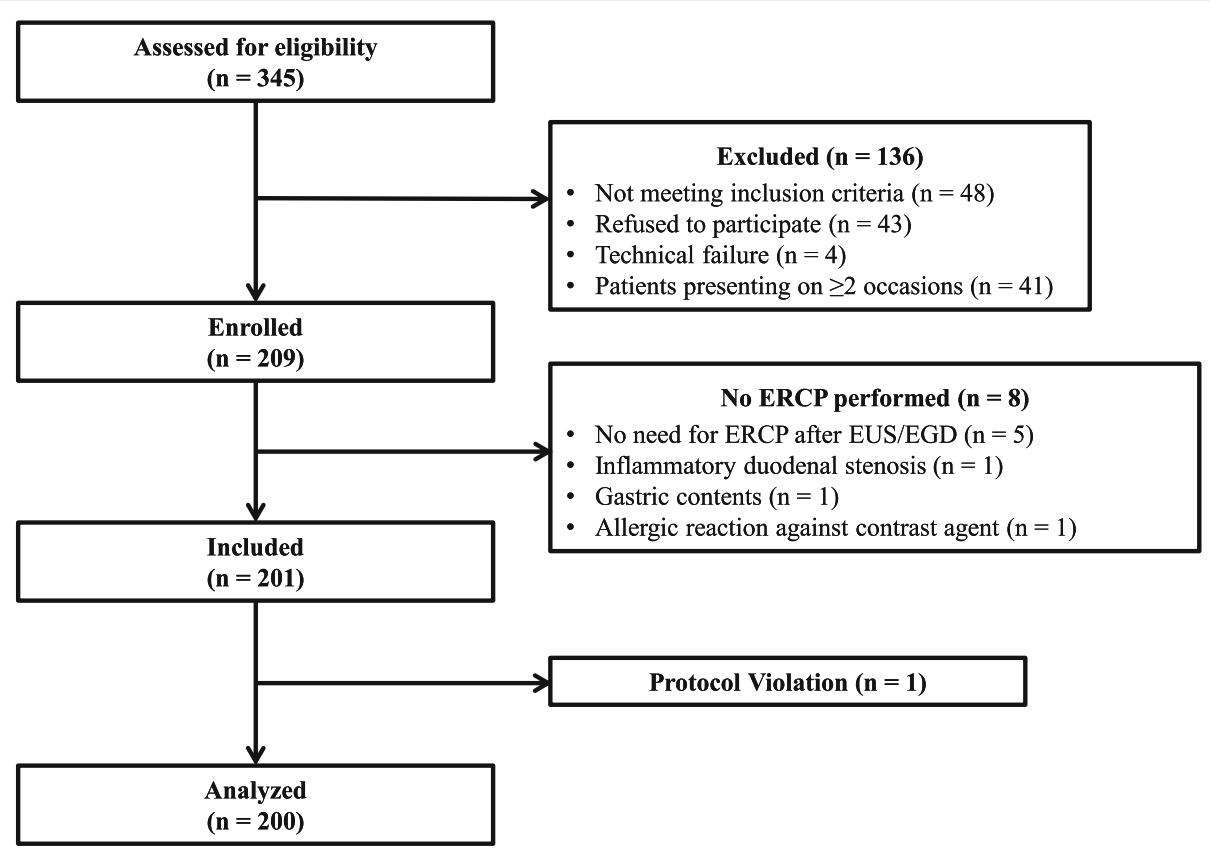

Fig. 1 Study flow chart, EGD: esophagogastroduodenoscopy, ERCP: endoscopic retrograde cholangiopancreatography, EUS: endoscopic ultrasound

Table 1 Characteristics of patients excluded from the study

\begin{tabular}{ll}
\hline Excluded patients (not meeting inclusion criteria) & $\mathrm{n}$ \\
\hline $\mathrm{O}_{2}$ sat $<90 \%$ & $\mathbf{4 8}$ \\
$\mathrm{SBP}<90 \mathrm{mmHg}$ & 7 \\
Lack of written informed consent & 3 \\
Age $<18$ years & 30 \\
Need for general anesthesia & 2 \\
Patients who refused to participate & 6 \\
Technical failure ${ }^{\text {a }}$ & $\mathbf{4 3}$ \\
Patients presenting on $\geq \mathbf{2}$ occasions ${ }^{\mathbf{b}}$ & $\mathbf{4}$ \\
No ERCP performed & $\mathbf{4 1}$ \\
No need for ERCP after EUS/EGD & $\mathbf{8}$ \\
Inflammatory duodenal stenosis & 5 \\
Gastric contents & 1 \\
Allergic reaction against the contrast agent & 1 \\
Protocol violation ${ }^{c}$ & 1 \\
Total &
\end{tabular}

EGD Esophagogastroduodenoscopy, ERCP Endoscopic retrograde cholangiopancreatography, EUS Endoscopic ultrasound, $\mathrm{O}_{2}$ sat Oxygen saturation, SBP Systolic blood pressure

${ }^{a}$ Technical failure refers to monitoring system failure such that vital parameters could not be completely and accurately recorded

batients who presented on $\geq 2$ occasions were included in the study only once. The most complete data set was chosen for evaluation. In cases of data sets that were identical with regard to recorded data, data were chosen randomly

cProtocol violation was observed in 1 patient who was accidentally administered additional drugs (different from those included in the study protocol) for sedation
Most patients were classified as ASA class III (59.5\%, $n=119 / 200)$ and class II $(36.5 \%, n=73 / 200)$. The most common underlying disease was a hepatic disorder in $63.5 \%$ of the patients $(n=127 / 200)$, and the most common indication for ERCP was malignant biliary stenosis in $58 / 200$ patients $(29 \%)$, followed by postoperative stricture at the biliary anastomosis after liver transplantation in $22.5 \%$ of the patients $(n=45 / 200)$. The mean total propofol dose administered for sedation was $287 \pm 134$ mg (range $80-800 \mathrm{mg}$ ) with a medication dosage of $3.8 \pm 1.7 \mathrm{mg} / \mathrm{kg}$ (range $1-10.3 \mathrm{mg} / \mathrm{kg}$ ). The mean total remifentanil dose administered was $135 \pm 68 \mu \mathrm{g}$ (range $20-500 \mu \mathrm{g})$ with a medication dosage of $1.8 \pm 1.0 \mu \mathrm{g} / \mathrm{kg}$ (range $0.3-6.4 \mu \mathrm{g} / \mathrm{kg}$ ).

\section{Cardiorespiratory data}

Cardiorespiratory parameters are presented in Table 3. We observed baseline $\mathrm{O}_{2}$ sat of $98.0 \pm 1.6 \%$. Desaturation (represented by $\mathrm{O}_{2}$ sat $<90 \%$ ) was recorded in 18 of 200 patients (9\%) during ERCP. Of these 18 patients, hypoxemia was recorded once in 11 patients $(61 \%)$ and twice in 5 patients $(28 \%)$ during the entire period of investigation. We observed 3 episodes of hypoxemia and 4 episodes of desaturation in 1 patient each $(5.5 \%)$ during the entire procedure. The mean $\mathrm{O}_{2}$ sat was $97.7 \pm 2.7 \%$ (range 65-100\%). Most hypoxemic complications were minor events, and patients were successfully treated with minor airway interventions (e.g., chin lift, jaw thrust, and/or increased oxygen supplementation via nasal cannula) and did not necessitate discontinuation of ERCP. ERCP was terminated in 2 patients secondary to 
Table 2 Patients' clinical and demographic characteristics, procedural indications, and medication dosages

\begin{tabular}{|c|c|}
\hline Male, n (\%) & $128 / 200(64)$ \\
\hline Mean age $\pm S D$, (years) & $56.3 \pm 15.2$ \\
\hline \multicolumn{2}{|l|}{ ASA score, n (\%) } \\
\hline । & $7(3.5)$ \\
\hline$\|$ & $73(36.5)$ \\
\hline$\|$ & $119(59.5)$ \\
\hline IV & $1(0.5)$ \\
\hline \multicolumn{2}{|l|}{ Disorder, n (\%) } \\
\hline Hepatic & $127 / 200(63.5)$ \\
\hline Gastrointestinal & $31 / 200(15.5)$ \\
\hline Postoperative & $20 / 200(10)$ \\
\hline Other disorders & $22 / 200(11)$ \\
\hline \multicolumn{2}{|l|}{ Procedural indications, n (\%) } \\
\hline Malignant jaundice & $58 / 200(29)$ \\
\hline Stricture after LTX & $45 / 200(22.5)$ \\
\hline Stones/sludge & $37 / 200(18.5)$ \\
\hline Postoperative complications & 18/200 (9) \\
\hline Other reasons & $42 / 200(21)$ \\
\hline \multicolumn{2}{|l|}{ Medication dosages } \\
\hline Propofol mean dose \pm SD, (mg) & $287 \pm 134$ \\
\hline Propofol mean dosage $\pm \mathrm{SD}$, (mg/kg) & $3.8 \pm 1.7$ \\
\hline Remifentanil mean dose $\pm \mathrm{SD},(\mu \mathrm{g})$ & $135 \pm 68$ \\
\hline Remifentanil mean dosage $\pm S D$, $(\mu \mathrm{g} / \mathrm{kg})$ & $1.8 \pm 1.0$ \\
\hline
\end{tabular}

ASA I: normal healthy patients, ASA II: patients with mild systemic disease, ASA III: patients with severe systemic disease, ASA IV: patients with severe systemic and life-threatening disease

ASA American Society of Anesthesiologists

LTX Liver transplantation, SD Standard deviation

sedation-related hypoxemia: 1 patient showed oxygen saturation decrease secondary to aspiration. However, $\mathrm{O}_{2}$ sat was rapidly restored to safe levels after termination of the ERCP by placing the patient in the supine position with assisted ventilation support via a respiratory mask. Spontaneous respiration was restored, and the patient was awake when transferred to the recovery unit with an $\mathrm{O}_{2}$ sat of $99 \%$ on 21 of oxygen supplementation via nasal cannula. ERCP was repeated 2 days later and was successfully performed under general anesthesia. ERCP was terminated in the second patient owing to apnea with recurrent decline in $\mathrm{O}_{2}$ sat to $<90 \%$ and agitation. $\mathrm{O}_{2}$ sat improved following increased oxygen supplementation via nasal cannula, and after recovery from sedation, spontaneous respiration was restored with an $\mathrm{O}_{2}$ sat of $99 \%$ without oxygen supplementation when the patient arrived at the recovery unit. The patient recovered completely from this sedation-related complication. ERCP was not repeated in this case because cholestasis resolved after the initial ERCP.
Table 3 Patients' cardiorespiratory data

\begin{tabular}{|c|c|}
\hline \multicolumn{2}{|l|}{$\mathrm{O}_{2}$ sat } \\
\hline Baseline $\mathrm{O}_{2}$ sat \pm SD (\%) & $98.0 \pm 1.6$ \\
\hline $\mathrm{O}_{2}$ sat $<90 \%, \mathrm{n}(\%)$ & 18/200 (9) \\
\hline$n=1, \mathrm{n}(\%)$ & $11 / 18(61)$ \\
\hline$n=2, n(\%)$ & $5 / 18(28)$ \\
\hline$n=3, n(\%)$ & $1 / 18(5.5)$ \\
\hline$n=4, n(\%)$ & $1 / 18(5.5)$ \\
\hline Apnea, n (\%) & $1 / 200(0.5)$ \\
\hline Mean $\mathrm{O}_{2}$ sat $\pm \mathrm{SD}$ (range), (\%) & $\begin{array}{l}97.7 \pm 2.7 \\
(65-100)\end{array}$ \\
\hline \multicolumn{2}{|l|}{ SBP } \\
\hline SBP baseline \pm SD, $(\mathrm{mmHg})$ & $142 \pm 21$ \\
\hline Mean SBP \pm SD (range), (mmHg) & $\begin{array}{l}128 \pm 24 \\
(74-220)\end{array}$ \\
\hline $\mathrm{SBP}<90 \mathrm{mmHg}, \mathrm{n}(\%)$ & $18 / 200(9)$ \\
\hline$n=1, \mathrm{n}(\%)$ & 7/18 (39) \\
\hline$n=2, n(\%)$ & 7/18 (39) \\
\hline$n=3, \mathrm{n}(\%)$ & $2 / 18(11)$ \\
\hline$n=4, n(\%)$ & $2 / 18(11)$ \\
\hline \multicolumn{2}{|l|}{ HR } \\
\hline Baseline HR \pm SD, (bpm) & $79 \pm 14$ \\
\hline Mean HR \pm SD (range), (bpm) & $\begin{array}{l}80 \pm 15 \\
(40-128)\end{array}$ \\
\hline Bradycardia, n (\%) & $0 / 200(0)$ \\
\hline $\begin{array}{l}\text { Termination of procedure secondary to adverse } \\
\text { events, } n(\%)\end{array}$ & $2 / 200(1)$ \\
\hline
\end{tabular}

The baseline SBP was $142 \pm 21 \mathrm{mmHg}$. Hypotension (SBP $<90 \mathrm{mmHg}$ ) occurred in 18 of 200 patients (9\%). Among these 18 patients, a single episode of hypotension was recorded in 7 patients (39\%) and 2 episodes of hypotension in 7 patients $(39 \%)$ during the entire period of investigation. More than 2 episodes of hypotension occurred in 4 patients during the entire procedure ( 3 episodes: $n=2 / 18,4$ episodes: $n=2 / 18$ ). The mean SBP was $128 \pm 24 \mathrm{mmHg}$ (range $74 \mathrm{mmHg}-$ $220 \mathrm{mmHg}$ ). Hypotension was successfully treated with intravenous fluid replacement in all patients, and no patient needed adrenergic drug administration. All procedures could be continued until completion, and all patients with hypotension recovered completely.

The mean heart rate was $79 \pm 14 \mathrm{bpm}$ (range 40-128 bpm). No patient developed bradycardia (heart rate $<40$ $\mathrm{bpm}$ ) during the investigation.

Sedation-related complications (hypotension and hypoxemia) occurred in 36 of 200 patients (18\%), and ERCP was terminated in 2 patients (1\%) secondary to sedationrelated hypoxemia. 
Cannulation rate and procedural data

As shown in Table 4, cannulation of the major duodenal papilla was performed successfully in all patients $(n=$ $200,100 \%)$. The mean duration of ERCP was $25 \pm 16$ min, with a maximum and minimum duration of 85 and $3 \mathrm{~min}$, respectively. The mean time for intubation of the major papilla was $7.00 \pm 6.5 \mathrm{~min}$ (range $1 \mathrm{~min}-53 \mathrm{~min}$ ).

\section{Post-anesthesia recovery data}

Post-anesthesia recovery data are shown in Table 5. We observed that $97 \%$ of the patients were awake and oriented upon arrival at the recovery unit $(n=194 / 200)$, and the mean recovery time after sedation was $14 \pm 10$ min. Based on the PARS tool, post-anesthesia recovery was assessed $30 \mathrm{~min}$ after completion of ERCP. As shown in Table $5,60 \%$ of the patients $(n=120 / 200)$ recovered completely with a maximum PARS value achieved $30 \mathrm{~min}$ after the investigation. The mean PARS value was $9.5 \pm 0.7$ (minimum of 7 to a maximum of 10 points).

All patients were able to breathe deeply and cough freely ( $n=200 / 200,100 \%)$. Regarding circulatory status, data were unavailable in 4 patients (for various reasons). SBP $\pm 20 \%$ of the pre-anesthetic level was observed in $67 \%$ of the patients $(n=131 / 196)$, and SBP $\pm 20-49 \%$ of the pre-anesthetic level was observed in $31 \%$ of the patients $(n=61 / 196) .94 .5 \%$ of the patients were completely awake after $30 \mathrm{~min}(n=189 / 200)$. Complete resumption of activity was observed in 186 of 200 patients (93\%), and normal skin color was restored in $99.5 \%$ of the patients $(n=199 / 200)$.

\section{Patient and endoscopist satisfaction}

As shown in Table 4, the mean VAS of the endoscopy team with regard to sedation quality was significantly

Table 4 Cannulation rate, procedural data and satisfaction in patients and endoscopists

\begin{tabular}{|c|c|}
\hline \multicolumn{2}{|l|}{ Cannulation } \\
\hline Successful cannulation, n (\%) & $200(100)$ \\
\hline Mean time for intubation $\pm S D$, (min) & $7 \pm 6.5$ \\
\hline Range, (min) & $1-53$ \\
\hline \multicolumn{2}{|l|}{ Procedural data } \\
\hline Mean duration $\pm S D$, (min) & $25 \pm 16$ \\
\hline Range, (min) & $3-85$ \\
\hline \multicolumn{2}{|l|}{ Endoscopist-reported satisfaction } \\
\hline Mean VAS $\pm S D$ & $9.3 \pm 1.3$ \\
\hline \multicolumn{2}{|l|}{ Patient-reported satisfaction } \\
\hline Mean VAS $\pm S D$ & $9.6 \pm 0.8$ \\
\hline $\begin{array}{l}\text { Willingness to undergo a repeat } \\
\text { procedure under the same conditions, n (\%) }\end{array}$ & 197/200 (98.5) \\
\hline
\end{tabular}

Table 5 Post-anesthesia recovery data

\begin{tabular}{ll}
\hline Post-anesthesia recovery data & \\
\hline PARS after 30 min, $n(\%)$ & $120 / 200(60)$ \\
10 & $60 / 200(30)$ \\
9 & $15 / 200(7.5)$ \\
8 & $5 / 200(2.5)$ \\
7 & $9.5 \pm 0.7$ \\
Mean PARS \pm SD & $14 \pm 10$ \\
Recovery time \pm SD, (min) & \\
Patients awake/oriented upon arrival at the recovery unit, & $194 / 200(97)$ \\
$n$ (\%) &
\end{tabular}

Activity, n (\%)

Able to move 4 extremities voluntarily or on command $186 / 200$ (93)

Able to move 2 extremities voluntarily or on command 14/200 (7)

Unable to move extremities voluntarily or on $\quad$ 0/198 (0) command

Respiration, $\mathbf{n}$ (\%)

Able to breathe deeply and cough freely 200/200

(100)

Dyspnea or limited breathing

$0 / 200(0)$

Apneic

$0 / 200(0)$

Circulation, $\mathrm{n}$ (\%)

$\mathrm{SBP} \pm 20 \%$ of pre-anesthetic level

131/196 (67)

$\mathrm{SBP} \pm 20-49 \%$ of pre-anesthetic level

$61 / 196(31)$

$\mathrm{SBP} \pm 50 \%$ of pre-anesthetic level

$4 / 196(2)$

Consciousness, $\mathbf{n}(\%)$

Fully awake

$189 / 200$

(94.5)

Arousable on calling

$11 / 200(5.5)$

Unresponsive

$0 / 200(0)$

Color, n (\%)

Normal 199/200

(99.5)

Pale, dusky, blotchy, jaundiced, or other 1/200 (0.5)

Cyanotic

$0 / 200(0)$

PARS Post Anesthesia Recovery Score, SBP Systolic blood pressure, SD Standard deviation

high (mean VAS: $9.3 \pm 1.3$ ). Patient satisfaction scores with regard to sedation quality were also high (mean VAS: $9.6 \pm 0.8$ ), and 197 of 200 patients were willing to undergo a repeat procedure under the same conditions (98.5\%).

\section{Mortality and morbidity data}

No sedation-related complications occurred during the 30 days follow-up period. Post-ERCP complications within 30 days occurred in 9 of 200 patients (4.5\%). Major complications were cholangitis, which occurred in 3 of 200 patients (1.5\%) and pain $(n=3 / 200,1.5 \%)$, followed by post-ERCP pancreatitis $(n=2 / 200,1 \%)$ and 
bleeding $(n=1 / 200,0.5 \%)$. Both patients ( 1 with mild and the other with moderate pancreatitis according to the Atlanta classification system) who developed postERCP pancreatitis recovered without any complications after standard therapeutic interventions.

The 30-day mortality rate after ERCP observed in this study was $1 \%$. Two patients died within 30 days of nonsedation-related causes: 1 patient developed acute coronary heart syndrome after surgery and the other died of cancer.

\section{Discussion}

To date, optimal sedation techniques for complex endoscopic procedures are controversial. Additionally, the safety and efficacy of anesthesiologist-administered sedation vs. sedation administered by non-anesthesia personnel remain unclear. Our large-scale prospective cohort study investigated the safety and efficacy of anesthesiologist-administered sedation for ERCP.

Results of non-anesthesiologist administered sedation of studies included in the Cochrane Review [7-11], and results of anesthesiologist-administered sedation of the present study are shown in Table 6 . The mean medication dose of propofol used in our study was lower than that used in studies included in the Cochrane Review, which described sedation administered by nonanesthesia personnel [7-11]. However, Goudra et al. [15] reported a lower mean propofol dosage in cases of sedation administered by non-anesthesia personnel, which however was associated with significantly low patient and endoscopist satisfaction. Reportedly, the quality of sedation administered by anesthesia personnel was good, although compared with our study, their study required significantly higher propofol doses [15]. In our study, propofol (a sedative without analgesic properties) complemented the action of remifentanil (an opioid analgesic) against ERCP-associated discomfort. The lower propofol doses used in our study are attributable to the synergistic anesthetic effect of these drugs administered to all patients [16].

The studies included in the Cochrane Review focused on propofol sedation administered by non-anesthesia personnel, and hypoxemia was the most common sedation-related complication (in $11-37 \%$ of the patients) [7-11]. Sedation-related hypoxemia occurred in only $9 \%$ of our patients. This is an unusual finding, particularly because we used a propofol-remifentanil combination for sedation, which should reduce the propofol dose required, thereby reducing the cardiocirculatory adverse events associated with propofol use, although the risk of respiratory events is higher. Our findings could be attributed to the fact that oxygen supplementation in patients led to safer sedation, but early desaturation was not detected in such cases. Kongkam et al. [8] and Vargo et al. [11] reported higher hypoxemia rates (22.4 and $37 \%)$ during sedation administered by non-anesthesia personnel without oxygen supplementation than those reported by studies in which patients received oxygen supplementation (11-11.8\%) [9, 10]. With regard to anesthesiologist-administered sedation, Berzin et al. reported a hypoxemia rate of $12.5 \%$ and sedation-related adverse events in $21 \%$ of cases [17]. Smith et al. also reported a hypoxemia rate of $9.5 \%$ and sedation-related adverse events in $19 \%$ of cases [18]. These findings concur with those of our study and indicate that sedation administered by anesthesia personnel is safer with regard to hypoxemia.

This result might also be attributed to anesthesiologists' skills to better manage sedation-related complications such as performing an adequate airway management (e.g. intubation) in case of hypoxemia.

Kongkam et al. reported a procedure termination rate of $4.5 \%$ secondary to sedation-related complications (agitation, aspiration, and apnea) with propofol sedation administered by non-anesthesia personnel [8]. In the study reported by Vargo et al., failure to complete the procedure was not recorded [11]. The procedure termination rate was $1 \%$ in our study, which was associated with sedation-related hypoxemia secondary to aspiration and apnea. Oxygen saturation returned to normal levels in both patients with minor airway interventions, followed by complete recovery. Most patients in our study (59.5\%) were classified as ASA class III, whereas most patients described by Vargo et al. and Kongkam et al. were classified as ASA classes I and II. The mean age of our patients was 56.3 years, which was comparable to the mean age of patients included in the studies reported by Kongkam et al. and Vargo et al. $[8,11]$. Buxbaum et al. reported a failure rate of $7 \%$ secondary to sedationrelated complications in cases of gastroenterologistadministered sedation and $1.3 \%$ in cases of sedation administered by anesthesia personnel [19], which concur with our findings. We observed that compared with patients receiving sedation administered by non-anesthesia personnel, those receiving sedation administered by anesthesia personnel showed a better safety profile and lower termination rate.

Hypotension occurred in $5.2-15.8 \%$ and bradycardia in $0.0-6.5 \%$ of the patients receiving propofol sedation administered by non-anesthesia personnel across studies included in the Cochrane Review [7-11]. Our study results concur with these findings. All patients with hypotension were successfully treated with intravenous fluid replacement, and no procedure was terminated secondary to cardiocirculatory complications. Berzin et al. and Smith et al. reported similar hypotension rates of $7.2-9.5 \%$ in patients undergoing anesthesiologistadministered sedation $[17,18]$. 
Table 6 Results of non-anesthesiologist administered sedation of previous studies and results of anesthesiologist-administered sedation of present study

\begin{tabular}{|c|c|c|c|c|c|c|}
\hline Studies & Number of patients & Age, years & Gender, $[\mathrm{m} / \mathrm{f}]$ & ASA & Procedure & $\begin{array}{l}\text { Mean propofol } \\
{[\mathrm{mg}]}\end{array}$ \\
\hline Vargo et al. [11] & 38 & $52.9 \pm 2.4$ & $21 / 17$ & $\begin{array}{l}\text { ASA I: } 14(36.8 \%) \\
\text { ASA II: } 16(42.1 \%) \text { ASA } \\
\text { III: } 8 \text { (21.1\%) } \\
\text { ASA IV: - }\end{array}$ & $\begin{array}{l}\text { EUS } \\
\text { ERCP }\end{array}$ & 356.8 \\
\hline Riphaus et al. [9] & 77 & $83.7 \pm 7.8$ & $35 / 42$ & $\begin{array}{l}\text { ASA I: - } \\
\text { ASA II: - } \\
\text { ASA III: } 32(41.6 \%) \\
\text { ASA IV: } 39(50.6 \%)\end{array}$ & ERCP & 322 \\
\hline Kongkam et al. [8] & 67 & $52.3 \pm 11.9$ & $40 / 27$ & $\begin{array}{l}\text { ASA I: } 26(38.8 \%) \\
\text { ASA II: } 22(32.8 \%) \\
\text { ASA III: } 19(28.4 \%) \\
\text { ASA IV: - }\end{array}$ & ERCP & 299.9 \\
\hline Schilling et al. [10] & 76 & 82.4 & $25 / 51$ & $\begin{array}{l}\text { ASA I: - } \\
\text { ASA II: - } \\
\text { ASA III: } 34(44.7 \%) \\
\text { ASA IV: } 12(15.8 \%)\end{array}$ & $\begin{array}{l}\text { ERCP } \\
\text { EUS } \\
\text { DBE }\end{array}$ & 376 \\
\hline Present study & 200 & $56.3 \pm 15.2$ & $128 / 72$ & $\begin{array}{l}\text { ASA I: } 7(3.5) \\
\text { ASA II: } 73(36.5) \\
\text { ASA III: } 119(59.5) \\
\text { ASA IV: } 1 \text { (0.5) }\end{array}$ & ERCP & 287 \\
\hline Studies & $\begin{array}{l}\text { Number of patients } \\
\text { with hypoxemia }\end{array}$ & $\begin{array}{l}\text { Number of patients } \\
\text { with hypotension }\end{array}$ & \multicolumn{2}{|c|}{$\begin{array}{l}\text { Number of patients with } \\
\text { bradycardia }\end{array}$} & \multicolumn{2}{|c|}{$\begin{array}{l}\text { Failure to complete } \\
\text { examination }\end{array}$} \\
\hline Vargo et al. [11] & $14(37 \%)$ & $6(15.8 \%)$ & \multicolumn{2}{|l|}{ NA } & \multicolumn{2}{|l|}{ NA } \\
\hline Riphaus et al. [9] & $8(11 \%)$ & $6(8 \%)$ & \multicolumn{2}{|l|}{$3(4 \%)$} & \multicolumn{2}{|l|}{ NA } \\
\hline Kongkam et al. [8] & $15(22.4 \%)$ & $6(9 \%)$ & \multicolumn{2}{|l|}{$2(3 \%)$} & \multicolumn{2}{|l|}{3} \\
\hline Schilling et al. [10] & $9(11.8 \%)$ & $4(5.2 \%)$ & \multicolumn{2}{|l|}{$5(6.5 \%)$} & \multicolumn{2}{|l|}{ NA } \\
\hline Present study & $18(9 \%)$ & $18(9 \%)$ & \multicolumn{2}{|l|}{$0(0 \%)$} & \multicolumn{2}{|l|}{$2(1 \%)$} \\
\hline Studies & Procedure time, [min] & $\begin{array}{l}\text { Endoscopist-reported } \\
\text { satisfaction }\end{array}$ & \multicolumn{2}{|c|}{ Patient-reported satisfaction } & \multicolumn{2}{|c|}{ Time to recovery, [min] } \\
\hline Vargo et al. [11] & $53.6 \pm 3$ & $8.17 \pm 0.28$ & \multicolumn{2}{|l|}{$9.01 \pm 0.3$} & \multicolumn{2}{|l|}{$18.6 \pm 6.5$} \\
\hline Riphaus et al. [9] & $29 \pm 19$ & $8.7 \pm 1.7$ & \multicolumn{2}{|l|}{$8.4 \pm 1.9$} & \multicolumn{2}{|l|}{$22 \pm 7$} \\
\hline Kongkam et al. [8] & $39.8 \pm 32.5$ & 7.4 & \multicolumn{2}{|c|}{ No significant difference } & \multicolumn{2}{|l|}{$17.24 \pm 5.99$} \\
\hline Schilling et al. [10] & $42 \pm 18$ & $7 \pm 2$ & \multicolumn{2}{|l|}{ NA } & \multicolumn{2}{|l|}{ NA } \\
\hline Present study & $25 \pm 16$ & $9.3 \pm 1.3$ & \multicolumn{2}{|l|}{$9.6 \pm 0.8$} & \multicolumn{2}{|l|}{$14 \pm 10$} \\
\hline
\end{tabular}

Bradycardia: heart rate < 40 bpm; DBE Double-balloon enteroscopy, EUS Endoscopic ultrasound; Hypotension: $\mathrm{SBP}<90 \mathrm{mmHg}$; Hypoxemia: $\mathrm{O}_{2}$ saturation < $90 \%$; NA: data not recorded

The cannulation rate was $100 \%$ in our study, and the mean procedure time was $25 \pm 16 \mathrm{~min}$, which is shorter than that observed in studies reporting sedation administered by non-anesthesia personnel (29-54 $\mathrm{min})$ [8-11], which was attributed to the more rapid and deeper sedation performed by anesthesiologists. Non-anesthesia personnel might be hesitant with regard to drug administration, and endoscopists usually accept suboptimal sedation quality when propofol is administered under their supervision. This hypothesis is supported by the fact that the endoscopist satisfaction rate was higher in procedures performed with sedation administered by anesthesia personnel [15].
The mean recovery time was $14 \pm 10 \mathrm{~min}$ in our study, and $97 \%$ of the patients were awake and oriented upon transfer to the recovery unit. The recovery time was slightly shorter compared with studies reporting sedation administered by non-anesthesia personnel (range $17.2-22 \mathrm{~min})[8,9,11]$. The mean PARS value was $9.5 \pm$ 0.7 , which was higher than that reported by Riphaus et al. [9] in a study describing propofol sedation administered by non-anesthesia personnel (mean PARS value $8.3 \pm 1.2$ ), indicating more rapid recovery in patients undergoing sedation administered by anesthesia personnel.

Previous studies have reported that compared with patients receiving "conventional sedation" (benzodiazepines 
alone or combined with opioids), patients receiving propofol sedation for ERCP showed higher post-procedural patient satisfaction $[9,20]$. Most patients (mean VAS score: $9.6 \pm 0.8$ ) and most endoscopists (mean VAS score: $9.3 \pm$ 1.3 ) in our study reported being "very satisfied" with the sedation quality. Similar results were reported by Goudra et al. (mean VAS [patients]: 9.8, mean VAS [endoscopists]: 9) in patients receiving sedation administered by anesthesia personnel; however, a significantly higher propofol dosage was used in the study [15]. Notably, the quality of sedation administered by non-anesthesia personnel was associated with low patient and endoscopist satisfaction (mean VAS [patients]: 7.2, mean VAS [endoscopists]: 6) [15]. These results concur with those of other studies describing low patient and endoscopist satisfaction associated with sedation administered by non-anesthesia personnel [8-11]. Compared with non-anesthesia personnel, anesthesiologists usually administer higher propofol doses, thereby achieving deeper sedation and better sedation quality reported by patients and endoscopists [15].

Reportedly, the ERCP-induced complication and mortality rates are 5-10 and $0.1 \%-1 \%$, respectively [1-5]. The most common post-procedural complication is postERCP pancreatitis (prevalence rate 1-15\%) [3, 21, 22]. Complication rates in our study were significantly low (4.5\%), and post-ERCP pancreatitis occurred in only $1 \%$ of patients. The mortality rate was $1 \%$; however, these patients died of non-sedation related events secondary to underlying disease. Lapidus et al. reported high safety and efficacy of endoscopist-administered balanced propofol sedation during ERCP without any adverse outcomes [23]. However, patients included in their study were classified as ASA classes I and II (indicating inclusion of a large percentage of low-risk patients). In concordance with this, the recently updated European Society of Gastrointestinal Endoscopy guidelines suggest "primary involvement of an anesthesiologist" to administer propofol in patients with ASA and/or Mallampati scores $\geq 3$ or in those with comorbidities predisposing to airway obstruction [24].

Limitations of this study included the uncontrolled design, which makes it difficult to compare outcomes of anesthesiologist-administered sedation with those of non-anesthesiologist-administered sedation. Nevertheless, discussed study results and performed comparisons between this study and various other studies might provide an indication in this context. Moreover, despite the large number of patients included in this study our data are only the results of a single-center study and it might not be possible to generalize our findings. Although this study has certain limitations, it does provide a start for future studies evaluating the role of anesthesia personnel in the administration of sedation regarding safety and efficacy for complex endoscopic procedures compared to sedation administered by non-anesthesia personnel. If the results of our study could be confirmed in larger multicenter studies with randomized controlled design, they could have an important impact on guidelines for sedation during complex interventional endoscopy.

\section{Conclusions}

We conclude that sedation administered by anesthesia personnel for ERCP is safe and is associated with a high rate of successful and rapid interventions, as well as short post-anesthesia recovery times and high patient and endoscopist satisfaction.

\section{Abbreviations \\ ASA: American Society of Anesthesiologists; ECG: Electrocardiography; EGD: Esophagogastroduodenoscopy; ERCP: Endoscopic retrograde cholangiopancreatography; EUS: Endoscopic ultrasound; FDA: US Food and Drug Administration; $\mathrm{O}_{2}$ sat: Oxygen saturation; PARS: Post Anesthesia Recovery Score; SBP: Systolic blood pressure; VAS: Visual analogue scale}

\section{Acknowledgements}

Not applicable.

\section{Authors' contributions}

CCZ: This author helped perform the data analysis, data interpretation and prepare and revise the manuscript. NG: This author helped design the conceptual design, acquire the data, and revise the manuscript. PK: This author helped revise and edit the manuscript. CB: This author helped revise and edit the manuscript. TB: This author helped revise and edit the manuscript. MAW: This author helped revise and edit the manuscript. PS: This author helped revise and edit the manuscript. AS: This author helped design the conceptual design, prepare and revise the manuscript. All authors read and approved the final manuscript.

\section{Funding}

None.

\section{Availability of data and materials}

The datasets used and analyzed during the current study are available from the corresponding author on reasonable request.

\section{Ethics approval and consent to participate}

Our study protocol conformed to the Declaration of Helsinki and was approved by the Ethics Committee of the University Hospital Heidelberg vide letter no S-457/2013. Written informed consent to participate was obtained from all patients included in the study.

\section{Consent for publication}

Not applicable.

\section{Competing interests}

The authors declare that they have no competing interests.

\section{Author details}

${ }^{1}$ Department of Gastroenterology, Heidelberg University Hospital, Im Neuenheimer Feld 410, 69120 Heidelberg, Germany. ${ }^{2}$ Department of Anesthesiology, Heidelberg University Hospital, Heidelberg, Germany. ${ }^{3}$ Department of General Surgery, Heidelberg University Hospital, Heidelberg, Germany. ${ }^{4}$ Department of Anesthesiology, GRN Hospital Schwetzingen, Schwetzingen, Germany.

Received: 1 November 2019 Accepted: 24 May 2020

Published online: 28 May 2020

\section{References}

1. Cohen S, Bacon BR, Berlin JA, Fleischer D, Hecht GA, Loehrer PJ Sr, McNair AE Jr, Mulholland M, Norton NJ, Rabeneck L, et al. National Institutes of Health state-of-the-science conference statement: ERCP for diagnosis and therapy, January 14-16, 2002. Gastrointest Endosc. 2002;56(6):803-9. 
2. Freeman ML. Adverse outcomes of ERCP. Gastrointest Endosc. 2002;56(6 Suppl):S273-82.

3. Vandervoort J, Soetikno RM, Tham TC, Wong RC, Ferrari AP Jr, Montes H, Roston AD, Slivka A, Lichtenstein DR, Ruymann FW, et al. Risk factors for complications after performance of ERCP. Gastrointest Endosc. 2002;56(5): 652-6.

4. Mariani A, Segato S, Anderloni A, Cengia G, Parravicini M, Staiano T, Tontin GE, Lochis D, Cantu P, Manfredi G, et al. Prospective evaluation of ERCP performance in an Italian regional database study. Dig Liver Dis. 2019;51(7): 978-84.

5. Talukdar R. Complications of ERCP. Best Pract Res Clin Gastroenterol. 2016; 30(5):793-805.

6. Riphaus A, Wehrmann T, Weber B, et al. S3-Leitlinie "Sedierung in der gastrointestinalen Endoskopie" 2008 (AWMF-Register-Nr. 021 / 014) [S3guidelines--sedation in gastrointestinal endoscopy]. Z Gastroenterol. 2008; 46(11):1298-330. https://doi.org/10.1055/s-2008-1027850.

7. Garewal D, Powell S, Milan SJ, Nordmeyer J, Waikar P. Sedative techniques for endoscopic retrograde cholangiopancreatography. CochraneDatabase Syst Rev. 2012;(6):CD007274. Published 2012 Jun 13. https://doi.org/10.1002/ 14651858.CD007274.pub2.

8. Kongkam $\mathrm{P}$, Rerknimitr $\mathrm{R}$, Punyathavorn $\mathrm{S}$, Sitthi-Amorn C, Ponauthai $\mathrm{Y}$, Prempracha N, Kullavanijaya P. Propofol infusion versus intermittent meperidine and midazolam injection for conscious sedation in ERCP. J Gastrointestin Liver Dis. 2008;17(3):291-7.

9. Riphaus A, Stergiou N, Wehrmann T. Sedation with propofol for routine ERCP in high-risk octogenarians: a randomized, controlled study. Am J Gastroenterol. 2005;100(9):1957-63.

10. Schilling D, Rosenbaum A, Schweizer S, Richter H, Rumstadt B. Sedation with propofol for interventional endoscopy by trained nurses in high-risk octogenarians: a prospective, randomized, controlled study. Endoscopy. 2009;41(4):295-8.

11. Vargo JJ, Zuccaro G Jr, Dumot JA, Shermock KM, Morrow JB, Conwell DL, Trolli PA, Maurer WG. Gastroenterologist-administered propofol versus meperidine and midazolam for advanced upper endoscopy: a prospective, randomized trial. Gastroenterology. 2002;123(1):8-16.

12. Aldrete JA. The post-anesthesia recovery score revisited. J Clin Anesth. 1995; 7(1):89-91.

13. Banks PA, Bollen TL, Dervenis C, Gooszen HG, Johnson CD, Sarr MG, Tsiotos GG, Vege SS. Acute pancreatitis classification working G: classification of acute pancreatitis-2012: revision of the Atlanta classification and definitions by international consensus. Gut. 2013;62(1):102-11.

14. Bradley EL 3rd. A clinically based classification system for acute pancreatitis. Summary of the international symposium on acute pancreatitis, Atlanta, Ga, September 11 through 13. Arch Surg 1993. 1992;128(5):586-90.

15. Goudra BG, Singh PM, Gouda G, Borle A, Gouda D, Dravida A, Chandrashakhara V. Safety of non-anesthesia provider-administered Propofol (NAAP) sedation in advanced gastrointestinal endoscopic procedures: comparative meta-analysis of pooled results. Dig Dis Sci. 2015 60(9):2612-27

16. Westmoreland CL, Hoke JF, Sebel PS, Hug CC Jr, Muir KT. Pharmacokinetics of remifentanil (GI87084B) and its major metabolite (Gl90291) in patients undergoing elective inpatient surgery. Anesthesiology. 1993;79(5):893-903.

17. Berzin TM, Sanaka S, Barnett SR, Sundar E, Sepe PS, Jakubowski M, Pleskow DK, Chuttani R, Sawhney MS. A prospective assessment of sedation-related adverse events and patient and endoscopist satisfaction in ERCP with anesthesiologistadministered sedation. Gastrointest Endosc. 2011;73(4):710-7.

18. Smith ZL, Mullady DK, Lang GD, Das KK, Hovis RM, Patel RS, Hollander TG, Elsner J, Ifune C, Kushnir VM. A randomized controlled trial evaluating general endotracheal anesthesia versus monitored anesthesia care and the incidence of sedation-related adverse events during ERCP in high-risk patients. Gastrointest Endosc. 2019;89(4):855-62.

19. Buxbaum J, Roth N, Motamedi N, Lee T, Leonor P, Salem M, Gibbs D, Vargo J. Anesthetist-directed sedation favors success of advanced endoscopic procedures. Am J Gastroenterol. 2017;112(2):290-6.

20. Jung M, Hofmann C, Kiesslich R, Brackertz A. Improved sedation in diagnostic and therapeutic ERCP: propofol is an alternative to midazolam. Endoscopy. 2000;32(3):233-8.

21. Masci E, Toti G, Mariani A, Curioni S, Lomazzi A, Dinelli M, Minoli G, Crosta C, Comin U, Fertitta A, et al. Complications of diagnostic and therapeutic ERCP: a prospective multicenter study. Am J Gastroenterol. 2001;96(2):417-23.
22. Freeman ML, DiSario JA, Nelson DB, Fennerty MB, Lee JG, Bjorkman DJ, Overby CS, Aas J, Ryan ME, Bochna GS, et al. Risk factors for post-ERCP pancreatitis: a prospective, multicenter study. Gastrointest Endosc. 2001; 54(4):425-34.

23. Lapidus A, Gralnek IM, Suissa A, Yassin K, Khamaysi I. Safety and efficacy of endoscopist-directed balanced propofol sedation during endoscopic retrograde cholangiopancreatography. Ann Gastroenterol. 2019;32(3):303-11.

24. Dumonceau JM, Riphaus A, Schreiber F, Vilmann P, Beilenhoff U, Aparicio JR, Vargo JJ, Manolaraki M, Wientjes C, Racz I, et al. Non-anesthesiologist administration of propofol for gastrointestinal endoscopy: European Society of Gastrointestinal Endoscopy, European Society of Gastroenterology and Endoscopy Nurses and associates guideline--updated June 2015. Endoscopy. 2015;47(12):1175-89.

\section{Publisher's Note}

Springer Nature remains neutral with regard to jurisdictional claims in published maps and institutional affiliations.

Ready to submit your research? Choose BMC and benefit from:

- fast, convenient online submission

- thorough peer review by experienced researchers in your field

- rapid publication on acceptance

- support for research data, including large and complex data types

- gold Open Access which fosters wider collaboration and increased citations

- maximum visibility for your research: over $100 \mathrm{M}$ website views per year

At BMC, research is always in progress.

Learn more biomedcentral.com/submissions 